\title{
Treatment of dysthyroid ophthalmopathy with local Guanethidine
}

\author{
J. STANLEY GANT, D. R. H. LEWIS, AND M. T. HARRISON \\ Tennent Institute of Ophthalmology, University of Glasgow, Scotland
}

The use of local adrenergic blocking agents in the treatment of dysthyroid ophthalmopathy is becoming accepted following the report of Sneddon and Turner (1966). Earlier work indicated that local adrenergic blocking agents were effective in the treatment of chronic simple glaucoma (Stepanik, I96I) after initial trials of systemic Guanethidine on normal and glaucomatous human eyes had pointed to the usefulness of adrenergic blocking agents in ophthalmic therapy (Keates, Krishna, and Leopold, I960). Oosterhuis (1962) used local Guanethidine drops successfully in the treatment of glaucoma, noting incidentally that there was a narrowing of the palpebral fissure, and several subsequent trials of local adrenergic blocking agents have since shown that of the available substances, including Bethanidine, Phentolamine, and Propanolol, Guanethidine is the most effective for local ophthalmic use.

The eye complications of hyperthyroidism can be resolved essentially into the three cardinal signs of dysthyroid ophthalmopathy, namely exophthalmos, upper lid retraction, and ophthalmoplegia, together with the less obvious signs which include infiltration of the eyelids, orbital oedema, and chemosis. Exophthalmos is the most serious of the cardinal signs as it can lead to corneal exposure with keratitis, and if progressive can even cause the loss of the eye, while ophthalmoplegia can be very disabling through the resulting diplopia. Both exophthalmos and ophthalmoplegia are relatively rare compared with upper lid retraction, which is a common finding during the hyperthyroid state and not uncommonly persists when the euthyroid state has been reached after treatment. In the ophthalmic endocrine clinic of the Tennent Institute of Ophthalmology it has been confirmed that most dysthyroid patients complaining of prominence of the eyes have either minimal exophthalmos or none, and that the apparent protrusion of the eyes is due to exposure of a rim of sclera above the cornea through upper lid retraction (Fig. IB). As this apparent prominence of the eyes can be a serious cosmetic blemish and is usually accompanied by a gritty feeling or frank discomfort, the reduction of upper lid retraction would clearly be of benefit to the patient. Unfortunately, although upper lid retraction may lessen with systemic treatment of the hyperthyroid state, it does not always do so, and until local adrenergic blocking agents were used no reputable form of treatment had been available. Before Sneddon and Turner (1966) published their report the only available treatment for upper lid retraction was either a surgical attempt to lower the eyelid by section of the levator palpebrae superioris muscle or by decompression of the orbit, or alternatively palliative optical treatment could be offered which included protective contact lenses or protective spectacles. Surgical treatment of upper lid retraction is clearly undesirable and optical remedies are generally ineffective and at best purely symptomatic; hence the introduction of Guanethidine has meant that for the first time residual upper lid retraction, a previously untreatable condition, might be effectively remedied. 
Before introducing Guanethidine to routine clinical use in patients with local eye signs accompanying thyroid disorders, it was resolved to carry out an extended trial of loca $\bar{B}$ Guanethidine eye drops on a large number of patients. Until the present, only short-term trials on small numbers of patients have been recorded (Sneddon and Turner, 1966 $\overrightarrow{\overline{\vec{c}}}$ Crombie and Lawson, 1967 ). This communication reports the results of the trial whick was conducted with the following aims:

(x) To assess the efficacy of local Guanethidine in the reduction of upper lid retraction

(2) To establish the strength of drops and dosage schedule necessary in the treatment of upper lid retraction.

(3) To determine if permanent improvement results from this treatment or if long ternw treatment is necessary.

(4) To assess the effect of local Guanethidine on exophthalmos and the other eye signs of dysthyroid ophthalmopathy, since some previous reports have indicated that there tends tof be an improvement in exophthalmos.

(5) To ascertain what local or systemic side-effects occur with long-term use.

\section{Method}

Patients with dysthyroid ophthalmopathy were considered for investigation, but only those whos $\vec{\wp}$ physical signs included upper eyelid retraction were admitted to the trial. 81 patients with upper eyelid retraction ( 14 males and 67 females) entered the trial, the average age on admission being $46 \cdot I$ years and the average duration of treatment with Guanethidine eye drops 54 weeks. All ths patients had been or were being treated for thyroid dysfunction at the time of admission to the tria and 77 were euthyroid both clinically and by the estimation of serum protein bound iodine; 29 og these euthyroid patients required no maintenance therapy and 48 were having replacement therapy? Only four patients were thyrotoxic at the time of admission to the trial; one became euthyroid afte a subtotal thyroidectomy and the other three were successfully treated with carbimazole.

Each patient was fully assessed on admission to the trial. Initially, a full ophthalmologica芦 examination was carried out, including standard photography with full face and lateral views to show the degree of upper eyelid retraction and exophthalmos, if present. Exophthalmos and eyeliक. retraction were both measured directly and were again measured from the standard photographs Similar complete assessment including photography was carried out after the initial response to treatment was obtained in each case. After this the patient was followed up at I or 2-weekly intervals, and when treatment had become established, at longer intervals.

When first establishing the efficacy of this treatment only cases of marked upper eyelid retraction? were chosen. These patients were admitted to hospital for detailed investigation, including photo graphic exophthalmometry, and were then treated with hourly instillations of Guanethidine sulphate Io per cent. drops in methyl cellulose for 8 hours. A further assessment was made and 48 hours later they were given 10 per cent. Guanethidine I drop four times daily and discharged on this therapy to be followed up at the Out-patient Clinic. After this initial group of ten patients had beenw treated, it was obvious that a satisfactory lid position could be produced by 8 hours' intensive therap疋 with Guanethidine drops. Some regression tended to occur during the 48 -hours interval, but in ale cases the satisfactory lid position was again obtained when the drops were resumed.

Having established the efficacy of this intensive regime and the absence of systemic side-effects with this therapy (Cant and Lewis, 1969), it was decided there was no further need to admit patientso to hospital and the next group were treated as out-patients, being started on ro per cent. Guane- $\overrightarrow{\mathbb{D}}$ thidine I drop four times daily, again with satisfactory results. Where a good response was obtained the frequency of instillation was reduced, and eventually the strength of drops was reduced to 5 oro 2 per cent. again with reduced frequency. 
It was found in all cases except one that the improved lid positions were maintained on the 5 per cent. eye drops, the one exception being a woman of 48 years who showed some regression on 5 per cent. drops and had to revert to ro per cent. drops. In view of these good results all further new patients were treated with 5 per cent. drops, initially I drop three times daily, and when this regime was found to be adequate a reduction in frequency again became possible. The final stage was reached when a group of fourteen new patients were treated initially with one 2 per cent. drop three times daily. All these patients showed improvement in the upper lid retraction but in three of them further improvement was produced by using 5 per cent. drops. Eventually, when the optimum lid position was obtained in each case, this was held by the lowest strength of drop available ( 2 or 5 per cent.) at the lowest possible frequency. Throughout the trial great care was taken to instruct the patient on the correct instillation of eye drops, as we were concerned not only with the response to treatment but with the possibility of local toxic effects.

\section{Results}

In considering the effect of the drops in treating upper lid retraction, it was found best to assess the position of the upper eyelid in relation to the corneo-scleral junction (limbus) with the eyes in the primary position. In the normal patient the lower border of the upper eyelid "cuts" the cornea about $2 \mathrm{~mm}$. below the limbus (Fig. IA), whereas with upper lid retraction the sclera above the upper limbus (Fig. IB) is exposed, in marked cases by more than $5 \mathrm{~mm}$. This is in contrast to the appearance in simple exophthalmos, where the sclera is usually exposed below the lower limbus; where exophthalmos and upper lid retraction co-exist, there is usually a complete rim of exposed sclera (Fig. IC).
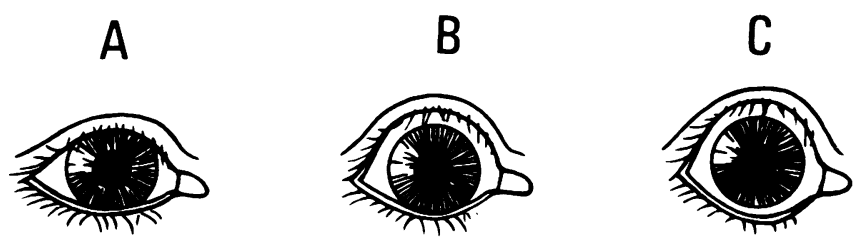

FIG. I Relationship of eyelids to eye

A Normal eyelid positions

B Scleral exposure above upper limbus caused by dysthyroid upper eyelid retraction

C A complete rim of sclera is visible due to exposure above limbus caused by upper eyelid retraction and exposure below limbus usually caused by exophthalmos

The presence of "scleral exposure" above the cornea is a reliable indication that there is retraction of the upper eyelid, but in marked or long-standing cases of upper lid retraction there tends to be some associated retraction of the lower eyelid and hence although scleral exposure below the cornea suggests that exophthalmos is present, it is not a reliable sign of this if upper lid retraction is also present. This lower lid retraction if present usually improves, together with upper lid retraction on treatment with Guanethidine and, whilst this adds still further to the improvement in the patient's appearance, this change in the position of the lower eyelid can create the false impression that a co-existing exophthalmos has also been successfully treated (Figs 2 and 3 overleaf).

The results of treatment were assessed as good if the upper eyelid margin came below the limbus, fair if the upper eyelid margin was at the limbus or left less than I $\mathrm{mm}$. of scleral exposure in the primary position, and poor if more than I $\mathrm{mm}$. of scleral exposure remained above the limbus but the position of the eyelid was better than before treatment. 


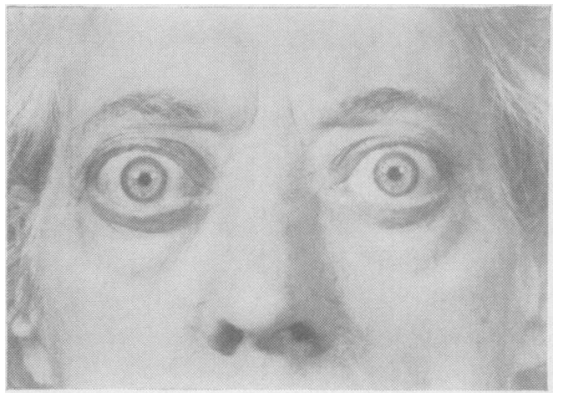

FIG. 2 Patient with bilateral upper eyelid retraction and associated lower eyelid retraction. There is no exophthalmos

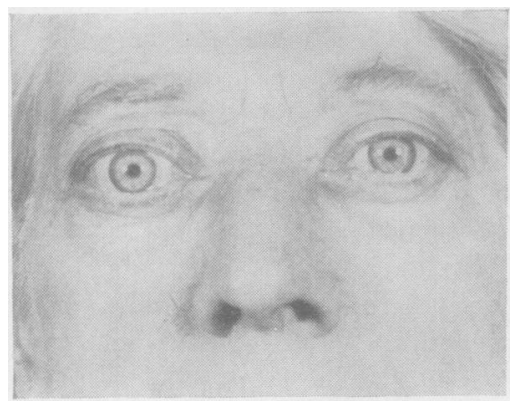

F I G. 3 Patient in Fig. 2 after treatment with Guanethidine drops

This extended trial showed Guanethidine drops to be effective in treating upper eyeliạ retraction in a large number of patients. The result of the trial is indicated in Table I $\mathrm{I}_{\mathrm{i}}^{+}$ this includes all 77 euthyroid patients and many are included several times under varyin regimes of therapy. The four thyrotoxic patients are not included in this Table as the were all under active systemic treatment, although all four showed an immediate response to the drops which were continued during treatment of the endocrine state.

Table I Response of upper eyelid retraction to various dosage schedules of local Guanethidine in 77 euthyroid patients

\begin{tabular}{|c|c|c|c|c|c|c|}
\hline \multirow{2}{*}{$\begin{array}{l}\text { No. of } \\
\text { patients }\end{array}$} & \multicolumn{2}{|l|}{ Therapy } & \multicolumn{4}{|c|}{ Response } \\
\hline & Guanethidine concentration & Dosage & Good & Fair & Poor & Nil \\
\hline IO & \multirow[t]{3}{*}{ Io per cent. } & Hrly for $8 \mathrm{hrs}$ & 7 & 3 & $\longrightarrow$ & - \\
\hline I 4 & & 4 times a day & 9 & 5 & - & - \\
\hline I 8 & & Twice daily & 13 & 5 & - & - \\
\hline 59 & \multirow[t]{4}{*}{5 per cent. } & 3 times a day & 42 & I 2 & 4 & I \\
\hline $4^{I}$ & & Twice daily & $3 \mathrm{I}$ & 9 & I & $-\ldots$ \\
\hline I I & & Daily & IO & I & $\cdot$ & $\cdots$ \\
\hline 5 & & Less than daily & 4 & I & $-\cdot$ & $\cdots$ \\
\hline 25 & \multirow[t]{4}{*}{2 per cent. } & 3 times a day & I 5 & 5 & 5 & - \\
\hline I 9 & & Twice daily & 14 & 4 & I & $\cdots$ \\
\hline 7 & & Daily & 5 & 2 & $-\ldots$ & $\cdots-$ \\
\hline 2 & & Less than daily & 2 & -- & - & $\longrightarrow$ \\
\hline
\end{tabular}

Almost all patients made a spontaneous comment on their improved appearance anc. relative freedom from the ocular discomfort or gritty sensation but many patients alsß mentioned that the eyes appeared to be red. This redness is due to the conjunctival vasor dilatation produced by local Guanethidine.

One of the aims of the trial was to determine the most effective strength of drops an $\Phi$ dosage schedule. Io per cent. drops were found to have an almost immediate effect abolishing upper lid retraction but this strength was abandoned when reduced strength were found to be almost equally effective. Io per cent. drops had previously been reported as causing superficial corneal disturbance and this was confirmed (Cant and Lewis, ig69) The rate of response varies from patient to patient and, although improvement tends t $\$$ appear sooner in recent rather than in long-standing cases, it is difficult to predict the rat 8 of improvement in the individual case. We feel it is best to start with 5 per cent. Guane 
thidine I drop three times daily until optimum improvement in the lid position is obtained and then to reduce the frequency to daily instillation if this is adequate and, if possible, to further reduce to instillation on alternate days. In most patients upper lid retraction was bilateral, but where it is unilateral, drops need to be instilled only on the affected side. As 5 per cent. drops are marginally more effective than 2 per cent., it seems best to confine the strength of drops to 5 per cent. and to control the effect by varying the frequency of administration. Most patients found it necessary to continue with the drops if permanent improvement was to be obtained, and in this respect the use of Guanethidine drops must be regarded as maintenance therapy. It was hoped that permanent improvement in lid position might be brought about after a prolonged period and hence the average duration of the trial was 54 weeks, but only seven of 77 euthyroid patients were able to discontinue the drops after several weeks of gradual reduction in therapy with no return to the former lid position. Most patients found that when therapy was reduced below a minimum level, upper eyelid retraction tended to recur, and if the drops were stopped for a few days the eyelids tended to resume the former position.

In contrast to early reports we found no change in the position of the eyes as measured by exophthalmometry. Thirty selected patients on varying schedules of administration had exophthalmometry performed at intervals with the results shown in Table II. The measurements were all made by the same observer, using a Leudde exophthalmometer. The mean readings in this group of patients show that no effect was produced on the position of the eyes. There was no improvement in muscle power in the patients who had ophthalmoplegia nor was there any improvement in chemosis or the other secondary signs of dysthyroid ophthalmopathy.

\section{Table II Exophthalmometry}

\begin{tabular}{|c|c|c|c|c|c|c|c|}
\hline \multirow{3}{*}{$\begin{array}{l}\text { No. of } \\
\text { patients }\end{array}$} & \multicolumn{2}{|l|}{ Therapy } & \multicolumn{4}{|c|}{ Mean exophthalmometry readings $(\mathrm{mm})}$. & \multirow{3}{*}{$\begin{array}{l}\text { No. with } \\
\text { clinical } \\
\text { exophthalmos }\end{array}$} \\
\hline & \multirow{2}{*}{$\begin{array}{l}\text { Guanethidine } \\
\text { concentration }\end{array}$} & \multirow{2}{*}{ Dosage } & \multicolumn{2}{|l|}{ Right } & \multicolumn{2}{|l|}{ Left } & \\
\hline & & & Before & $\begin{array}{l}\text { After } 2 \\
\text { months }\end{array}$ & Before & $\begin{array}{l}\text { After } 2 \\
\text { months }\end{array}$ & \\
\hline 10 & Io per cent. & Twice daily & $23 \cdot 2$ & $23 \cdot 4$ & $22 \cdot 6$ & $22 \cdot 7$ & 2 \\
\hline I0 & 5 per cent. & 3 times a day & $22 \cdot 8$ & $23 \cdot 0$ & $22 \cdot 3$ & $22 \cdot 1$ & I \\
\hline ro & 2 per cent. & 3 times a day & $22 \cdot 5$ & $22 \cdot 6$ & $22 \cdot 9$ & $22 \cdot 9$ & 3 \\
\hline
\end{tabular}

The final aim of the trial was to ascertain whether the drops produced any side-effect. No systemic side-effects were found and the local side-effects or unwanted pharmacological effects are recorded in a separate communication (Cant and Lewis, 1969).

\section{Discussion}

This extended trial in a large group of patients shows that Guanethidine drops are most effective in treating dysthyroid upper eyelid retraction. It must be emphasized that the effect is confined to improvement in the position of the eyelids alone and that there is no effect on the other signs of dysthyroid ophthalmopathy and that, in particular, exophthalmos is unchanged. Guanethidine may be considered to produce a medically-induced Horner's syndrome, and it is disappointing that there is no relative enophthalmos in the 
exophthalmic patients corresponding to the enophthalmos of this syndrome. Althoug the mechanism of production of dysthyroid ophthalmopathy is not understood, there little doubt that upper lid retraction is due to spasm of the levator palpebrae superioris muscle through either a humoral or local nervous mechanism and that the drops produe their effect by an adrenergic neuron-blocking mechanism. It might be inferred that the effect of Guanethidine in abolishing or reducing dysthyroid upper lid retraction confirn the theory that this lid retraction is due to spasm of the levator muscle and, although th $\frac{\Phi}{55}$ seems to be the case, the reservation must be made that the effectiveness of this drug when used in pharmacological doses does not necessarily confirm the theory.

Upper eyelid retraction is by far the commonest component of dysthyroid ophthalm $\vec{\circ}$ pathy and in dysthyroid patients the tendency is for the position of the eyelids to improve्E with treatment of the endocrine state. The majority of our patients were former thyrotoxic but had persistent upper lid retraction when in the euthyroid state. Suci patients with persistent upper lid retraction tend to show a spontaneous but slow improvê ment in lid position, but Guanethidine drops produced an almost immediate improvemert which could not be expected spontaneously. It might be argued that with the tendency spontaneous improvement, treatment with a local adrenergic-blocking agent is unnecessar but many of these patients develop ophthalmic complications due to corneal exposure and some may be disabled through keratitis and ocular infection, which are secondary to upp lid retraction. Guanethidine drops provide a useful alternative to leaving the patient to await a spontaneous remission or to the use of relatively ineffective palliative local ant biotic therapy or optical protective devices.

\section{Summary}

Local Guanethidine drops were used in 81 patients suffering from dysthyroid upper liâ retraction. They were found to be effective in reducing or abolishing upper lid retractio in almost all cases and 5 per cent. drops three times daily are suggested as initial therap\$ with a gradual reduction to a maintenance dosage. The drops were found to be ineffective in reducing exophthalmos or ophthalmoplegia.

We are grateful to Dr. A. K. Pittman and Dr. D. A. Richards of Ciba Laboratories Ltd., for making Guane thidine drops available to us during this trial and for help in the preparation of the paper.

\section{References}

CANT, J. S., and LEWIS, D. R. H. (1969) Brit. 7. Ophthal., 53, 239

CROMBIE, A. L., and LAWSON, A. A. H. (I967) Brit. med. J., 4, 592

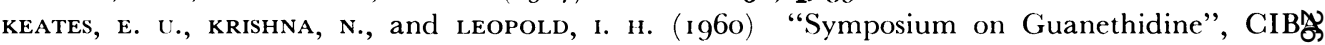

Summit, New Jersey, p. 66

oosterhuis, J. A. (I962) Arch. Ophthal. (Chicago), 67, 802

SNEDDON, J. M., and TURNER, P. (I966) Lancet, 2, 525

stepanik, J. (I96I) Klin. Mbl. Augenheilk., 139, I 74 